\title{
Stage 2 Neuroblastoma
}

National Cancer Institute

\section{Source}

National Cancer Institute. Stage 2 Neuroblastoma. NCI Thesaurus. Code C6639.

Stage 2 includes stage 2A and stage 2B. Stage 2A: Localized tumor with incomplete gross excision; representative ipsilateral nonadherent lymph nodes negative for tumor microscopically. Stage 2B: Localized tumor with or without complete gross excision, with ipsilateral nonadherent lymph nodes positive for tumor. Enlarged contralateral lymph nodes must be negative microscopically. (cancer.gov) 